\title{
Viable embryos and normal calves after nuclear transfer into Hoechst stained enucleated demi-oocytes of cows
}

\author{
M. E. Westhusin*, M. J. Levanduski, R. Scarborough, C. R. Looney and \\ K. R. Bondioli \\ Granada Bioscience Inc., 100 Research Parkway, Suite 100, College Station, TX 77840, USA
}

\begin{abstract}
Summary. Bovine oocytes were bisected, stained with Hoechst 33342 and observed under a fluorescent microscope to identify nucleated and enucleated demi-oocytes. Other oocytes were bisected but not stained, or bisected and only half of each oocyte stained, and viewed under a fluorescent microscope. The oocytes were then used for nuclear transfer by fusing them with embryonic blastomeres from a 5-6 day bovine embryo. The fusion rate and proportion developing into compact morulae or blastocysts was compared among different types of demi-oocytes. Expt 1 examined the effect of staining and indicated no effect on either fusion rate or embryonic development whether or not the oocytes were stained. In Expt 2, stained and unstained nucleated and enucleated oocytes were compared. As in the first experiment, there were no differences between stained and unstained demi-oocytes. There was no difference between fusion rates of nucleated and enucleated oocytes. However, there was a significant difference in embryonic development between nucleated $(10.4 \%)$ and enucleated $(22.6 \%)$ demi-oocytes $(P<0.05)$. In a final experiment, stained and unstained enucleated oocytes were used for nuclear transfer and the resulting embryos transferred into recipient cows. There was no difference in pregnancy rates or in the number of normal calves born whether stained or unstained recipient oocytes were used. Results from these experiments indicate that Hoechst staining and fluorescent microscopy can be used to identify enucleated demi-oocytes, and that these can be used for nuclear transfer, and result in viable embryos and normal calves. In these experiments, there was no difference between oocyte halves in their ability to develop normally following nuclear transfer whether or not they were stained with Hoechst 33342 and viewed with a fluorescent microscope. A greater number of viable embryos resulted after nuclear transfer when enucleated versus nucleated demi-oocytes were used as recipient ova.
\end{abstract}

Keywords: nuclear transfer; cow; Hoechst stain; embryo cloning; embryo/oocyte; micromanipulation

\section{Introduction}

The production of large numbers of identical bovine offspring by nuclear transfer offers the potential for tremendous genetic and economic gain for livestock agriculture. Use of a breeding programme involving the production of genetically superior identical animals would result in much greater genetic progress than is possible with standard programmes involving artificial insemination and embryo transfer (Nicholas \& Smith, 1983; Robl \& Stice, 1989). Another benefit of producing identical animals is that performance and production costs could be predicted more

\footnotetext{
*Present address: Department of Physiology \& Pharmacology, College of Veterinary Medicine, Texas A\&M University College Station, TX 77843-4466, USA.
} 
accurately by decreasing the great variability among animals. Accurate studies of interactions between genotype and environment are possible when using identical animals. Fewer animals would also be required for experiments: Robl \& Stice (1989) reported that, for traits such as milk production, one member of an identical pair could replace more than 20 random animals in an experiment.

Procedures used for nuclear transfer are currently too costly and inefficient to justify large-scale commercial use of the techniques. One of the major problems contributing to the inefficiency of nuclear transfer in the bovine embryo has been that of enucleating unfertilized oocytes. It is impossible to visualize the metaphase chromatin in unfertilized bovine oocytes when standard light microscopy is used. Enucleation of oocytes has therefore generally been accomplished by blind aspiration of the cytoplasm and is only 50-60\% successful (Prather et al., 1987; Robl \& Stice, 1989; Bondioli et al., 1990). Procedures for enucleation of bovine oocytes result in transfer of blastomere nuclei into nucleated oocytes approximately $50 \%$ of the time. Willadsen (1986) reported that blastomeres fused to nucleated oocytes form blastocysts at a very low rate. These embryos were probably tetraploid and it is unlikely that they would have developed to term.

Chromatin in mammalian oocytes and early embryos can be visualized easily with a fluorescent microscope when they are stained with Hoechst 33342 (Critser \& First, 1986; Luttmer \& Longo, 1986; Conover \& Gwatkin, 1988; Tsunoda et al., 1988). This fluorescent dye specifically binds to the adenine and thymine bases of DNA. Excessive stain concentration and prolonged exposure to ultraviolet light during observation by microscope may inhibit development (Critser \& First, 1986; Tsunoda et al., 1988). However, Tsunoda et al. (1988) used fluorescent staining and nuclear transfer in mouse embryos to demonstrate differential sensitivity of nuclei and cytoplasm to Hoechst staining and ultraviolet irradiation. Results indicated that cytoplasm of mouse zygotes was much more resistant to UV irradiation after Hoechst staining, and fluorescent microscopy could be used to visualize and remove metaphase chromatin without affecting the early developmental capability of the cytoplasm of the oocyte. These enucleated oocytes were used as recipients for nuclear transplantation of inner cell mass nuclei and a significant proportion resulted in development to the blastocyst stage (Tsunoda et al., 1988).

The aim of the present study was to determine if staining with Hoechst 33342 and examination with fluorescence microscopy could be used to identify enucleated bovine oocytes without interfering with embryonic development after nuclear transfer.

\section{Materials and Methods}

\section{Donor embryos and recipient oocytes}

Collection of donor embryos and recipient oocytes was as previously described (Bondioli et al., 1990; Westhusin et al., 1991). Briefly, mature crossbred and dairy-type cows were superovulated with a 4-day regimen of injections of follicle-stimulating hormone twice a day (FSH-P; Burns Biotec Inc., Omaha, NE). Total FSH-P administered for crossbred cows was $28 \mathrm{mg}$ and for dairy-type cows $37 \mathrm{mg}$. In addition to the fifth and sixth injection of FSH-P, $25 \mathrm{mg}$ prostaglandin $\mathrm{F}_{2 \alpha}$ (PGF2 $\alpha$, Lutalyse: UpJohn Inc., Kalamazoo, MI) was administered.

For collection of donor embryos, cows were artificially inseminated and the embryos collected on day $5-6$ of the oestrous cycle (oestrus $=$ day 0 ). Dulbecco's phosphate buffered saline supplemented with $2 \%$ fetal calf serum (FCS; Gibco, Grand Island, NY) was used as the collection medium. After isolation, the embryos were placed in phosphate buffered saline containing $1000 \mathrm{mg}$ D-glucose $1^{-1}, 36 \mathrm{mg}$ sodim pyruvate $\mathrm{I}^{-1}$ (Gibco, Grand Island, NY), $0 \cdot 4 \%$ bovine serum albumin (BSA; Sigma, St Louis, MO), and 1\% penicillin/streptomycin (10000 iu penicillin $\mathrm{G}$ sodium $\mathrm{ml}^{-1}$ and $10000 \mu \mathrm{g}$ streptomycin $\mathrm{ml}^{-1}$, Gibco, Grand Island, NY), (MPBS), and held for $1-3 \mathrm{~h}$ until they were used for nuclear transfer. In some cases, the donor embryos were frozen and thawed before being used for nuclear transfer (Westhusin et al., 1991). For collection of recipient oocytes, cows received an injection of 4000 iu human chorionic gonadotrophin (hCG; Phoenix Pharmaceuticals, St Joseph, MO) approximately $48 \mathrm{~h}$ after the first prostaglandin injection. Thirty-six to forty hours after hCG injection, the oviducts were surgically removed and oocytes recovered by flushing the oviducts with MPBS. The oocytes were isolated and held for $\mathrm{I}-3 \mathrm{~h}$ at room temperature until required.

\section{Nuclear transplantation}

Procedures for nuclear transfer were essentially those previously described (Willadsen, 1986; Bondioli et al., 1990; Westhusin et al., 1991). Oocytes were placed in a Petri dish containing MPBS supplemented with $5 \mu \mathrm{g}$ cytochalasin B 
$\mathrm{ml}^{-1}$ (Sigma Inc., St Louis, MO) and a slit was made in the zona pellucida extending approximately two-thirds of the circumference of the oocyte. A glass pipette was then used to remove approximately half of the oocyte cytoplasm and place it into another zona pellucida that had previously been slit and cleaned. The bisected oocytes were then placed in another Petri dish containing the donor embryo in fresh MPBS. The zona pellucida of the donor embryo was slit as described for oocytes. The embryonic cells were removed from the embryo one at a time with a micropipette approximately the same size as the blastomeres, and they were placed under the zona pellucida of the recipient oocyte halves so that the membranes of the oocyte and blastomere were in contact.

Electrofusion was used to transfer the nuclei of the embryonic blastomeres into the oocyte halves. The oocyte halves with blastomeres were equilibrated in Zimmerman's cell fusion medium (Amsco, Erie, PA) and transferred into a drop of fusion medium contained on a fusion chamber composed of two wire electrodes mounted on a Plexiglas slide $200 \mu \mathrm{m}$ apart. The demi-oocyte and embryonic blastomere were aligned by an a.c. of $600 \mathrm{kHz}, 6 \mathrm{~V}$, for $10 \mathrm{~s}$, after which fusion was initiated by applying three pulses of $15 \mathrm{~V}$ d.c. for $50 \mu \mathrm{s}$, each pulse $0.1 \mathrm{~s}$ apart. After fusion, the oocyte halves containing the blastomeres were transferred into fresh MPBS with $20 \%$ FCS supplemented in place of $\mathrm{BSA}$, and placed in an incubator at $37^{\circ} \mathrm{C}$. After $1 \mathrm{~h}$, the demi-oocytes were removed from the incubator and observed to determine how many had been successfully fused with blastomeres. All demi-oocytes, whether fused or not, were then embedded in a double layer of agar (Willadsen, 1979) and surgically transferred into ligated sheep oviducts. After 6 days the embryos were surgically recovered by flushing the sheep oviducts with MPBS and evaluated.

Experiment 1. In Expt 1, bovine demi-oocytes were assigned randomly to a control or treatment group. Oocytes in the control group were bisected and used for nuclear transfer as described above. Oocytes in the treatment group were bisected and incubated in $5 \mu \mathrm{g}$ Hoechst $33342 \mathrm{ml}^{-1}$ (bisbenzimide trihydrochloride, Sigma, St Louis, MO) in MPBS for $30 \mathrm{~min}$ at $37^{\circ} \mathrm{C}$. After staining, the demi-oocytes were rinsed in fresh MPBS for 5-10 min then viewed in $1 \mathrm{ml}$ nunc wells (Nunc Inc., Naperville, IL) at $400 \times$ magnification with a Zeiss ICM405 inverted microscope equipped with epifluorescence, Zeiss filter set 487701-02 (excitation $365 \mathrm{~nm}$ and emission $420 \mathrm{~nm}$, Zeiss, Oberkochen, Germany), and an Osram (HBO-50) superpressure mercury lamp. The demi-oocytes were viewed for approximately $10 \mathrm{~s}$ and the metaphase chromatin identified. After viewing, the demi-oocytes, both nucleated and enucleated, were used for nuclear transfer.

Experiment 2. In Expt 2, bovine oocytes were bisected and half of the cytoplasm removed and incubated in $5 \mu \mathrm{g}$ Hoechst $33342 \mathrm{ml}^{-1}$ for $30 \mathrm{~min}$ at $37^{\circ} \mathrm{C}$, while the other half was kept separately in MPBS. Stained demi-oocytes were rinsed in fresh MPBS at $37^{\circ} \mathrm{C}$ for 5-10 min, then viewed in $1 \mathrm{ml}$ nunc wells filled with fresh MPBS with a Nikon Labophot microscope equipped with epifluorescence, filters BA420 and EX365 (excitation $365 \mathrm{~nm}$ and emission $420 \mathrm{~nm}$, Nikon Inc., Houston, TX), an Osram (HBO-100) mercury short arc lamp and a 50\% neutral density filter. The demi-oocytes were viewed at $200 \times$ magnification to determine if they contained the metaphase chromatin (for less than $10 \mathrm{~s}$ ). Stained demi-oocytes with observed metaphase chromatin were considered nucleated and their nonstained reciprocal halves were presumed enucleated. Stained demi-oocytes with no observable chromatin were also considered enucleated and their nonstained reciprocal halves were presumed nucleated. This resulted in four different recipient oocyte types which represented four treatments: (i) stained enucleated, (ii) unstained enucleated, (iii) stained nucleated and (iv) unstained nucleated demi-oocytes. These were used for nuclear transfer.

Experiment 3. For Expt 3, a field trial was conducted over a period of 5 months in which stained and unstained enucleated demi-oocytes were obtained as described in Expt 2 and used for nuclear transfer. The resulting embryos were transferred into synchronized recipient cows on or about day 6 after nuclear transfer. Pregnancy rates, determined by rectal palpation at approximately 60 days of gestation, and the number of calves surviving to term were recorded.

\section{Analysis of data}

For Expts 1 and 2, the number of demi-oocytes successfully fused with blastomeres and the number developing into compact morulae or blastocysts (viable) were converted to a percentage. The average fusion rate and average percentage that were viable were then compared between treatments using Analysis of Variance (ANOVA). For Expt 3, count data were generated and the pregnancy rate and number of calves surviving to term compared using $\chi$-square analysis.

\section{Results}

The average percentage of demi-oocytes successfully fused with embryonic blastomeres was not different between the stained group (84.6\%) and the control group (82.3\%) (Table 1). There was also no difference in the proportion of embryos that developed into compact morula or blastocyst which, for controls, was $13 \cdot 6 \%$ and for the stained group $14.3 \%$.

No difference in any treatment group was indicated for fusion rate (Table 2). There was also no difference in embryonic development following nuclear transfer whether or not the recipient 
Table 1. Fusion rates and embryonic development after nuclear transfer into Hoechst stained and unstained bovine demi-oocytes

\begin{tabular}{lcccc}
\hline Treatment & $\begin{array}{c}\text { Experimental } \\
\text { repetitions }\end{array}$ & $n$ & $\begin{array}{c}\text { Average } \\
\text { fusion rate }\end{array}$ & $\begin{array}{c}\text { Average } \\
\text { per cent viable }\end{array}$ \\
\hline Stained & 14 & 159 & 84.6 & 14.3 \\
Unstained & 14 & 135 & $82 \cdot 3$ & 13.6 \\
\hline
\end{tabular}

No significant differences within columns; $P<0.05$ (ANOVA).

aiable: compact morula or blastocyst when collected from the sheep.

demi-oocytes were stained with Hoechst. Stained enucleated demi-oocytes resulted in the highest percentage development to compact morula or blastocyst $(25.6 \%)$ and was significantly different from both stained $(10 \cdot 8 \%)$ and unstained $(10 \cdot 0 \%)$ nucleated demi-oocytes. Unstained enucleated demi-oocytes resulted in the second highest percentage viable $(19.6 \%)$ but the difference between other treatments was not significant. When data from Expt 2 were pooled to evaluate the effect of staining independent of whether demi-oocytes were nucleated or not, and the effect of enucleation independent of whether they were stained or not, the only significant difference between treatments was that between enucleated and nucleated demi-oocytes, with enucleated demi-oocytes resulting in a greater number of viable embryos following nuclear transfer (Table 3). Hoechst staining had no effect on any parameter measured (Table 4).

Table 2. Fusion rates and embryonic development after nuclear transfer into Hoechst stained and unstained, nucleated and enucleated demi-oocytes

\begin{tabular}{lcccc}
\hline Treatment & Repetitions & $n$ & $\begin{array}{c}\text { Average } \\
\text { fusion rate }\end{array}$ & $\begin{array}{c}\text { Average } \\
\text { per cent viable }\end{array}$ \\
\hline Stained enucleated & 11 & 113 & $69 \cdot 8^{\mathrm{b}}$ & $25 \cdot 6^{\mathbf{b}}$ \\
Unstained enucleated & 11 & 114 & $70 \cdot 0^{\mathrm{b}}$ & $19 \cdot 6^{\mathbf{b c}}$ \\
Stained nucleated & 11 & 113 & $57 \cdot 5^{\mathrm{b}}$ & $10 \cdot 8^{\mathrm{c}}$ \\
Unstained nucleated & 11 & 119 & $63 \cdot 7^{\mathrm{b}}$ & $10 \cdot 0^{\mathrm{c}}$ \\
\hline
\end{tabular}

${ }^{a}$ Viable: compact morula or blastocyst when collected from the sheep.

${ }^{b c}$ Differences in superscripts within the same column denote a significant difference, $P<0.05$ (ANOVA).

Table 3. Fusion rates and embryonic development after nuclear transfer into nucleated and enucleated demi-oocytes

\begin{tabular}{lcccc}
\hline Treatment & Repetitions & $n$ & $\begin{array}{c}\text { Average } \\
\text { fusion rate }\end{array}$ & $\begin{array}{c}\text { Average } \\
\text { per cent viable }\end{array}$ \\
\hline Enucleated & 22 & 227 & $69 \cdot 9^{\mathrm{b}}$ & $22 \cdot 6^{\mathrm{b}}$ \\
Nucleated & 22 & 232 & $60 \cdot 6^{\mathrm{b}}$ & $10 \cdot 4^{\mathrm{c}}$ \\
\hline
\end{tabular}

aViable: compact morula or blastocyst when collected from the sheep.

${ }^{b c}$ Differences in superscripts within the same column denote a significant difference, $P<0.05$ (ANOVA).

The results of Expt 3 are given in Table 5. Neither pregnancy rate nor number of calves born depended on whether recipient demi-oocytes for nuclear transfer were Hoechst stained. 
Table 4. Fusion rates and embryonic development after nuclear transfer into Hoechst stained and unstained demi-oocytes

\begin{tabular}{lcccc}
\hline Treatment & Repetitions & $n$ & $\begin{array}{c}\text { Average } \\
\text { fusion rate }\end{array}$ & $\begin{array}{c}\text { Average } \\
\text { per cent viable }\end{array}$ \\
\hline Stained & 22 & 226 & $63 \cdot 7$ & $18 \cdot 2$ \\
Unstained & 22 & 233 & 66.9 & $14 \cdot 8$ \\
\hline
\end{tabular}

No significant differences within columns; $P<0.05$ (ANOVA).

${ }^{a}$ Viable: compact morula or blastocyst when collected from the sheep.

Table 5. Pregnancy rates and calving rates following nuclear transfer into Hoechst stained and unstained enucleated demi-oocytes

\begin{tabular}{lccc}
\hline Treatment & $\begin{array}{c}\text { Embryo } \\
\text { transfers }\end{array}$ & $\begin{array}{c}\text { Pregnancy } \\
\text { rate } \\
(\%)\end{array}$ & $\begin{array}{c}\text { Live } \\
\text { calves } \\
(\%)\end{array}$ \\
\hline Stained & 71 & $16(22 \cdot 5)$ & $15(93 \cdot 8)$ \\
Unstained & 123 & $23(18 \cdot 7)$ & $22(95 \cdot 7)$ \\
\hline
\end{tabular}

No significant differences within columns; $P<0.05$ ( $\chi$ square analysis).

\section{Discussion}

Results of these experiments demonstrate that Hoechst staining and fluorescent microscopy can be used to identify enucleated bovine demi-oocytes that can be successfully used as recipient ova for nuclear transfer. With the procedures described, there was no difference in the developmental capability of recipient demi-oocytes whether or not they were stained. The data did indicate that there was a distinct advantage in using enucleated demi-oocytes rather than nucleated demioocytes. Enucleated demi-oocytes resulted in a greater number of viable embryos following nuclear transfer than did nucleated demi-oocytes. Tsunoda (1988) reported that Hoechst staining and fluorescent microscopy could be used to visualize and remove metaphase chromatin from mouse oocytes. These enucleated oocytes were then used as recipient ova for nuclei of inner cell mass (ICM) cells; $23 \%$ developed to two-cell embryos and 5\% developed to blastocyst. The developmental rate of unstained enucleated mouse oocytes receiving ICM cells is unknown since this experiment was not conducted. However, in studies using pronuclear transfer, Tsunoda (1988) showed that the cytoplasm could withstand up to $40 \mathrm{~s}$ of irradiation following Hoechst staining before development began to decrease. It took only $12 \mathrm{~s}$ on average to visualize and remove metaphase chromatin, so it is doubtful whether staining had an effect on the developmental capability of the oocyte cytoplasm. Development to the blastocyst stage following nuclear transfer into enucleated mouse oocytes was less than reported here for cattle. This is likely to be due to differences between species. Previous work with mice has resulted in very limited development following transfer of embryonic nuclei into enucleated zygotes or oocytes when compared with other species (McGrath \& Solter, 1983, 1984; Surani et al., 1987). Another possibility is that donor nuclei were from a more advanced stage of development than those used in the present study, i.e. ICM cells versus cells from a 5-6 day morula. Work with amphibians has shown a decrease in development following nuclear transfer as more advanced embryos are used for nuclear donors (DiBerardino, 1987). However, Smith \& Wilmut (1989) reported 56\% development to blastocyst after transfer of sheep ICM cells to enucleated oocytes. 
Approximately $10 \%$ of the embryos produced by nuclear transfer into nucleated demi-oocytes resulted in embryos that developed to the morula or blastocyst stage. This value is similar to that reported by Willadsen $(1986,9 \cdot 3 \%)$ in sheep. It is unknown at this time if these embryos can produce a normal pregnancy and live calf. To date, we have transferred only seven such embryos into recipient cows and none has resulted in a pregnancy. When the oocyte staining techniques described here were applied in a commercial nuclear transfer programme, the pregnancy rate with enucleated demi-oocytes was 145 of $693(20.9 \%)$ compared with 23 of $138(16.7 \%)$ when both nucleated and enucleated demi-oocytes were used. This finding is consistent with that predicted if none, or at least very few, of the embryos produced following nuclear transfer(s) into a nucleated recipient oocyte resulted in pregnancy. These data do not exclude the possibility that some of these embryos could result in pregnancy and normal calves. It is possible that the oocyte might selfenucleate leaving only the transferred nucleus to direct development, or the oocyte chromatin may degenerate and/or disperse throughout the cytoplasm and not become involved in development (Bromhall, 1975).

This research was supported by private funding from Granada BioSciences, Inc. The authors gratefully acknowledge John Williams of Texas A \& M University for his assistance with the data analysis.

\section{References}

Bondioli, K.R., Westhusin, M.E. \& Looney, C.R. (1990) Production of identical bovine offspring by nuclear transfer. Theriogenology 33, 165-174.

Bromhall, J.D. (1975) Nuclear transplantation in the rabbit egg. Nature 258, 719-721.

Conover, J.C. \& Gwatkin, R.B.L. (1988) Pre-loading of mouse oocytes with DNA-specific fluorochrome (Hoechst 33342) permits rapid detection of spermoocyte fusion. Journal of Reproduction and Fertility 82, 681-690.

Critser, E.S. \& First, N.L. (1986) Use of a fluorescent stain for visualization of nuclear material in living oocytes and early embryos. Stain Technology 61, 1-5.

DiBerardino, M.A. (1987) Genomic potential of differentiated cells analyzed by nuclear transplantation. American Zoologist 27, 623-644.

Luttmer, S.J. \& Longo, F.J. (1986) Examination of living and fixed gametes and early embryos stained with supravital fluorochromes (Hoechst 33342 and 3,3'dihexyloxacarbocyanine iodide). Gamete Research $15,267-283$.

McGrath, J. \& Solter, D. (1983) Nuclear transplantation in the mouse embryo by microsurgery and cell fusion. Science 220, 1300-1302.

McGrath, J. \& Solter, D. (1984) Inability of mouse blastomere nuclei transferred to enucleated zygotes to support development in vitro. Science 226, 1317-1319.

Nicholas, F.W. \& Smith, C. (1983) Increased rates of genetic change in dairy cattle by embryo transfer and splitting. Animal Production 36, 341-353.

Prather, R.S., Barnes, F.L., Sims, M.M., Robl, J.M., Eyestone, W.H. \& First, N.L. (1987) Nuclear trans- plantation in the bovine embryo: assessment of donor nuclei and recipient oocyte. Biology of Reproduction 37, 859-866.

Robl, J.M. \& Stice, S.L. (1989) Prospects for commercial cloning of animals by nuclear transplantation. Theriogenology 31, 75-84.

Smith, L.C. \& Wilmut, I. (1989) Influence of nuclear and cytoplasmic activity on the development in vivo of sheep embryos after nuclear transplantation. Biology of Reproduction 40, 1027-1035.

Surani, M.A.H., Barton, S.C. \& Norris, M.L. (1987) Experimental reconstruction of mouse eggs and embryos: and analysis of mammalian development. Biology of Reproduction 36, l-16.

Tsunoda, Y., Shioda, Y., Onodera, M., Nakamura, K. \& Uchida, T. (1988) Differential sensitivity of mouse pronuclei and zygote cytoplasm to Hoechst staining and ultraviolet irradiation. Journal of Reproduction and Fertility 82, 173-178.

Westhusin, M.E., Pryor, J.H. \& Bondioli, K.R. (1991) Nuclear transfer in the bovine embryo: A comparison of 5-day, 6-day, frozen-thawed, and nuclear transfer donor embryos. Molecular Reproduction and Development 28, 119-133.

Willadsen, S.M. (1979) A method for culture of micromanipulated sheep embryos and its uses to produce monozygotic twins. Nature 277, 298-300.

Willadsen, S.M. (1986) Nuclear transplantation in sheep embryos. Nature 320, 63-65.

Received 28 March 1991 